\title{
Logical Homologies between Housting Prices Dynamics and Damped Harmonic Oscillations
}

\author{
M. BefeJ ${ }^{a *}$, S. Kulesza ${ }^{b}$ And R. Cellmer ${ }^{a}$ \\ ${ }^{a}$ University of Warmia and Mazury in Olsztyn, Department of Spatial Analysis and Real Estate \\ Market, ul. Prawocheńskiego 15, 10-720 Olsztyn, Poland \\ ${ }^{b}$ University of Warmia and Mazury in Olsztyn, Faculty of Mathematics and Computer Science, \\ ul. Stoneczna 54, 10-710 Olsztyn, Poland \\ Doi: 10.12693/APhysPolA.138.89 \\ *e-mail: miroslaw.belej@uwm.edu.pl
}

\begin{abstract}
The aim of the article is to employ the model of a damped harmonic oscillator (DHO) and critically damped harmonic oscillator (CDHO) to study the time-dependent evolution of time series of housing prices. The study treats the housing markets as complex, adaptive dynamic systems, which develop through synergistic network responses. This assumption is based on an observation of the synergies of the trends in housing prices, despite principal differences in the geospatial attributes of the markets under study. The logical homologies between housing prices dynamics and nonlinear harmonic oscillator is drawn from observation that a steep rise in housing prices (2006-2007) is, in fact, a transient disturbance to otherwise stable price evolution and is followed by an asymptotic return to a long-term equilibrium in a series of oscillations (system relaxation). Using the model of damped oscillator, it has been shown that the housing markets are highly inert. This can be seen during periods of rapid changes, when they quickly undergo deep structural transformations, although the pace of changes during longterm evolution is slow enough to keep the systems close to the state of equilibrium. The delay in the time series relative to each other indicates the dominant role of the Warsaw market as the initiator of changes and suggests the existence of links between markets, which supports the concept of local housing markets as a network of communicating vessels.
\end{abstract}

topics: harmonic oscillations, long-term equilibrium, homologies, housing market

\section{Introduction}

Housing is a special type of commodity because is a spatially immobile, highly durable, highly expensive, multidimensionally heterogeneous and physically modifiable commodity. These characteristics shape attitudes and behaviours toward housing and, in turn, influence neighborhood characteristics, mortgage markets, national housing policies and urban growth and decline [1]. Interconnections of housing development and the economy indicate that the former plays a significant role in elevating the level of social, economic and spatial cohesion of the country [2-4]. In a traditional approach (classical mainstream economy), the housing market is treated as a synonym for stability and safety and as a sphere which, to a significant degree, remains independent of the current economic situation in other markets, whose dynamics are incommensurate with the long-term and stable dynamics of the housing market. However, the traditional manner of understanding the housing market has been significantly redefined by the considerable intensification of the tendency for a close relation between housing markets and financial markets via the mortgage system and properly constructed financial instruments, which became apparent at the beginning of twenty-first century. These issues are presented in more detail in the work: Yates [5], Moulton [6], Siemińska [7], Yoshino [8], Chatterjee [9].

The consequence of such processes was the global financial crisis, sometimes known as the mortgage crisis, which significantly revised the manner of thinking about the housing market and was the cause of great interest in non-classic economic theories as an alternative to a mainstream economics that was unable to deal with the crisis phenomenon. According to Stiglitz [10] in the new paradigm of economics, classic concepts of demand and supply should be supplemented with problems resulting from information asymmetry and the role of state institutions in overcoming them. Additionally, he drew attention to the necessity of using evolutionary models (instead of models focused on aiming for the accomplishment of equilibrium) which, in turn, requires taking into account historical phenomena and observations on the dynamics of examined processes. Thus, in the changing face of mainstream economics it is widely recognized that the housing market has roots in current microeconomic and macroeconomic reality but 
is additionally subjected to the information asymmetries of market participants, state institutional influence, participants' behaviour, and a large variety of external stimuli. According to Jakimowicz and Kulesza [11] if the system's sensitivity threshold to external stimuli is exceeded, it becomes unstable and moves from a nearly stable state to a state that is far from equilibrium. These dynamic processes often induce key changes in the system's trajectory of evolution, leading to the transformation of one form into another in the process of discontinuous and discrete changes in the system's state. In such cases, in heterodox economic theory, the term "multiple equilibrium state" is used. Such systemic evolution from one equilibrium state to another, through deep structural transformations, is called an economic traverse or phase transition. In such a process, the state of stable equilibrium may go into a state of unstable equilibrium in the process of searching for a new stable state. Thus, during sudden changes, within a short time a new equilibrium is sought.

Such processes have been observed in many countries, including Poland, where a sudden increase in housing prices in 2006-2007 was followed by a longterm steady fall, with some fluctuations (oscillations, in a physical sense). After Poland's accession to the European Union in 2004, previously stable housing prices began to change slightly. From 2006 to 2007 , the annual housing price growth rate exceeded $100 \%$. Such a rapid increase was unique in the 25-year history of the Polish housing market. Beginning in 2008, housing prices started to drop by $3 \%$ to $6 \%$ a year. We believe that through turbulent price movements the housing market reached a new equilibrium level.

In this paper we assume, that the housing markets are complex, open and adaptive dynamic systems, which develop through synergistic network responses. Due to the variability of stimuli, in long periods housing markets remain in stable states, and only incidentally turn instable, which can be seen as an ultimate urge to find a new, alternative path of development due to exhaustion of resources for current evolution. This assumption is based on an observation of the synergism of trends in series of housing prices [12-15], which generates a correlation in trends of housing prices despite fundamental differences in their geospatial attributes.

This study aims to find out a logical homologies between damped harmonic oscillations (DHO) and critically damped harmonic oscillations (CDHO) and housing (dwellings) prices dynamics on the local housing markets (HM). This study examined the synergistic processes taking place in housing markets taking into account time series for dwellings prices in four selected cities of Poland regarded as the major centers in their regions: Warszawa (Mazovia - central Poland), Gdańsk (Pomerania northern Poland), Kraków (Małopolska — southern Poland) and Poznań (Wielkopolska — western Poland).

\section{Nonlinear damped harmonic motion as the logical homologies of long-term evolution of housing prices}

When comparing different systems, such as housing markets and oscillations in physical systems, we can use the concept of analogy or homology. In the simplest sense, the analogy is a comparison of two otherwise unlike things based on resemblance of a particular aspect. Logical homology, on the other hand, is a much broader concept than the superficial similarities between the phenomena studied in different fields. We deal with logical homology when the factors influencing given phenomena or processes are different, while the formal laws that govern the dynamics of seemingly different objects are homogeneous. According to Jakimowicz [16] the discovery of homology significantly facilitates research, since it accelerates obtaining the final explanation for phenomena. The notion of system, assigned a relatively broad meaning, enables the transfer of models from one discipline to the other.

The homology between housing market and nonlinear damped harmonic motion stems from the fact that sharp step in the prices can be thought of as a perturbation of otherwise stable evolution and is followed by long-term return to equilibrium in a series of oscillations (relaxation process) [17]. We used in our research a damped harmonic oscillations (DHO) and critically damped harmonic oscillations (CDHO) to describe the evolution of prices on the local housing markets. This kind of models is well fitted to the data consisting of at least two fundamentally different processes (rapid growth and asymptotic decrease).

Consider the mass-spring system and suppose that the mass is subject to a frictional damping force that opposes its motion and is directly proportional to its instantaneous velocity. For a system that has a small amount of damping, the period and frequency are nearly the same as for simple harmonic motion, but the amplitude gradually decreases over time. The solution of the damped harmonic motion (DHO) takes the form:

$$
\begin{gathered}
y(t)=y_{0}+\exp \left(-\frac{t-t_{0}}{\tau}\right)\left[A \cos \left(\frac{2 \pi}{T}\left(t-t_{0}\right)\right)\right. \\
\left.+B \sin \left(\frac{2 \pi}{T}\left(t-t_{0}\right)\right)\right],
\end{gathered}
$$

where $y_{0}$ - free term (equilibrium level), $t$ - time, $t_{0}$ - delay shift, $\tau$ - relaxation (decay) constant, $T$ - oscillation period (economic cycle), $A$ and $B$ model constants depending on the initial conditions.

Case in which damping of an oscillator causes it to return to equilibrium with the amplitude gradually decreasing to zero is referred to as underdamping. System returns to equilibrium faster but overshoots and crosses the equilibrium position at least once. 
Main statistical descriptors. Source: Central Statistical Office (GUS).

TABLE I

\begin{tabular}{c|c|c|c|c}
\hline \hline Town & Area $\left[\mathrm{km}^{2}\right]$ & Population & $\begin{array}{c}\text { Population density } \\
{\left[\text { people } / \mathrm{km}^{2}\right]}\end{array}$ & $\begin{array}{c}\text { Budget expenditure } \\
\text { per capita [EUR] }\end{array}$ \\
\hline Warszawa & 517 & 1.715 .517 & 3317 & 1764 \\
Gdańsk & 262 & 460.427 & 1758 & 1306 \\
Kraków & 327 & 758.463 & 2321 & 1315 \\
Poznań & 262 & 550.742 & 2103 & 1357
\end{tabular}

Critical damping is a special case of damped oscillatory motion defined as the condition in which the damping of an oscillator results in it returning as quickly as possible to its equilibrium position. The critically damped system may overshoot the equilibrium position, but if it does, it will do so only once. The solution of the critically damped harmonic motion (CDHO) takes the form:

$$
y(t)=y_{0}+\exp \left(-\frac{t-t_{0}}{\tau}\right)\left[A\left(t-t_{0}\right)+B\right]
$$

where $A$ and $B$ - model constants depending on the initial conditions.

Delay shift defines the beginning of the evolution of a system in an arbitrarily chosen timeframe looking for the sharp change in the state of a system. The system evolves with elapsed time regardless of being observed or not, hence the beginning of the time series cannot be taken as the absolute time. Similar $t_{0}$ values correspond to highly concurrent time series, in which sharp changes occur simultaneously, whereas the smallest delay among the series under study points at the market in a leading position (the trigger). Any violent event in that market propagates through the network and triggers similar events in other markets. This suggests that local housing markets form a system of communicating vessels.

Relaxation time $\tau$ corresponds to the time interval in which an observable decreases by $\left(\mathrm{e}^{-1}\right)$ with respect to its initial value. The smaller $\tau$ value, the smaller system inertia (faster return to the equilibrium state). In case of the housing markets under study, those with lower $\tau$ values turn out more sensitive to changes in outer circumstances.

Finally, free term $y_{0}$ refers to the equilibrium level of time series of the housing prices defining longterm values approached during asymptotic relaxation of the system.

\section{Results and discussion}

The search for similarity between time series of housing prices (average quarter-based prices per $1 \mathrm{~m}^{2}$ of dwellings on secondary market) in four Polish cities and nonlinear damped harmonic motion was conducted in the interval from 2006 till 2018. The time period was chosen so that it covered an interval of sharp changes in housing prices and their subsequent relaxation to the equilibrium state. Geographically, the selected cities are the local centers of their regions: Warszawa (Mazovia -
TABLE II

Statistical descriptors of the time-series Q3 2006-Q4 2018.

\begin{tabular}{l|c|c|c|c}
\hline \hline & Warszawa & Gdańsk & Kraków & Poznań \\
\hline$N_{\text {total }}$ & 50.00 & 50.00 & 50.00 & 50.00 \\
mean & 7848.92 & 5553.48 & 6197.52 & 5271.69 \\
std. dev. & 676.92 & 665.92 & 376.83 & 501.23 \\
skewness & 0.09 & 0.31 & 0.62 & -1.19 \\
kurtosis & -0.61 & 1.16 & 1.68 & 4.50 \\
coeff. of var. & 0.09 & 0.12 & 0.06 & 0.10 \\
mean absol. dev. & 565.91 & 503.94 & 278.36 & 343.13 \\
minimum & 6232.00 & 3926.00 & 5193.00 & 3332.05 \\
median & 7854.93 & 5551.68 & 6156.10 & 5241.02 \\
maximum & 9137.00 & 7499.40 & 7309.00 & 6356.71
\end{tabular}

central Poland), Gdańsk (Pomerania — northern Poland), Kraków (Małopolska — southern Poland) and Poznań (Wielkopolska - western Poland). Table I shows the main statistical descriptors for the selected towns.

The time series of dwellings, from Q3 2006 to Q4 2018, were taken from the Housing Prices Database maintained by the National Bank of Poland (NBP). Depending on the research field, time series are generated by random variables sampled at a wide range of periods, extending from a fraction of a second to years of observations. The housing market, which is fundamentally different from the financial market studied mainly in econophysics, allows time series of no less than a month to a quarter. Another specificity of this market is the time lag between the decision to purchase a dwelling and its acquisition (signing of a notary deed). This sometimes takes a few weeks. The average number of monthly sales of flats in medium-sized Polish cities does not exceed 150 (in some months it may even be fewer than 30). Therefore, minute, hourly, daily or weekly time series (as in the financial market) have no place in the housing market. Another problem of analysing time series in the housing market is that in each subperiod the average price is based on a completely independent (relative to the previous period) and heterogeneous set of housing transactions. Hence, there are significant differences in average prices between sub-periods. In the financial market, in each subsequent sub-period the same company (shares) or group of companies (index) is analysed. Table II presents the basic descriptive statistics of time series (average quarter-based prices per $1 \mathrm{~m}^{2}$ of dwellings on secondary market) for the examined cities. 
TABLE III

Dynamical properties of housing market obtained through fit of time-series of housing prices to damped harmonic motion model (DHO).

\begin{tabular}{c|c|c|c|c|c}
\hline \hline Town & $t_{0}$ [quaters] & $\tau$ [quaters] & $T$ [quaters] & $y$ [PLN] & $R^{2}$ \\
\hline Warszawa & 608 & 2792 & 3842 & 767406 & 0.728 \\
Gdańsk & 6.06 & 6.11 & 3508 & 545321 & 0.604 \\
Kraków & 5.69 & 428 & 1707 & 613823 & 0.558 \\
Poznań & 531 & 5.72 & 1404 & 530468 & 0.704 \\
\hline
\end{tabular}

The procedure of fitting of all time-series of housing prices to damped harmonic motion model was reached, for chi-square with value of $10^{-9}$.

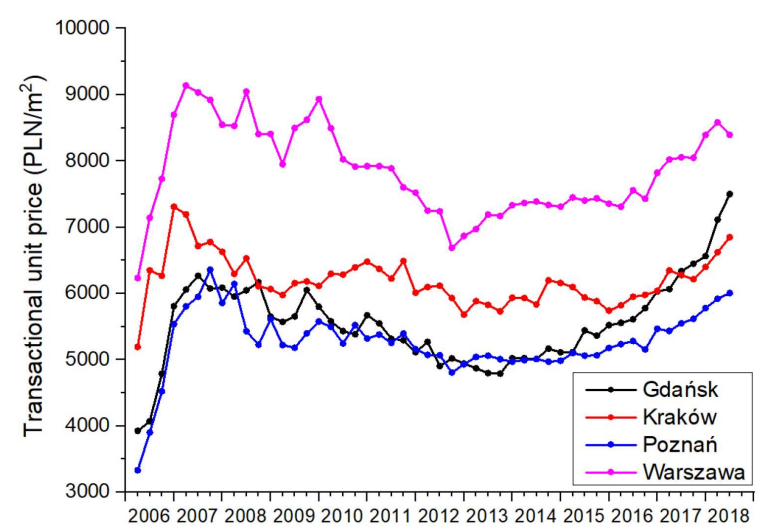

Fig. 1. Time series of the average quarter-based prices per $1 \mathrm{~m}^{2}$ of dwellings on secondary market in Warszawa, Gdańsk, Kraków and Poznań (2006-2018).

Figure 1 shows the time series of the average quarter-based prices per $1 \mathrm{~m}^{2}$ of dwellings on secondary market and in Warszawa, Gdańsk, Kraków and Poznań. Figure 2 shows box chart and histograms of the time series under analysis.

Figure 1 shows a strong synergy of price dynamics for dwellings between each examined Polish city. In 2006, an almost homogeneous reaction in prices is observed. The rapid increases in apartment prices ended in 2007 and were followed by a synergistic shift in trends and long-term steady declines in prices. Such a combination of two opposite states of housing price dynamics was the reason for the interest in non-linear models derived from physics (the damped harmonic motion model and the critically damped harmonic motion model). The homogeneous response of local housing markets suggests that local economic and demographic factors did not have a significant impact on the price dynamics. We believe that the almost homogeneous response of all studied housing markets creates network connections between them and determines the levels of synergy, time delays, and changes in the hierarchical structure between cities.

In the research, the damped harmonic oscillations model (DHO) and critically damped harmonic oscillations model (CDHO) were used to fit four time series of housing prices. Such models work best when the data consist of at least two processes
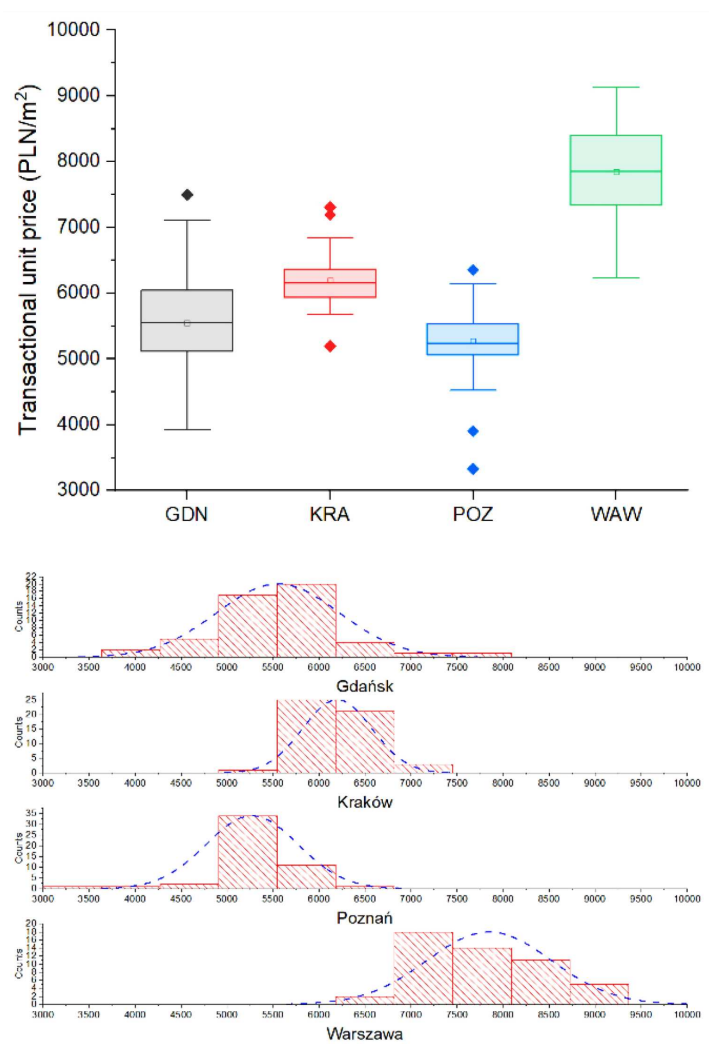

Fig. 2. Box plot (top) and histogram plot (bottom) of time series of the average quarter-based prices per $1 \mathrm{~m}^{2}$ of dwellings on secondary market in Warszawa, Gdańsk, Kraków and Poznań (2006-2018).

of fundamentally different characteristics (rapid vs. slow changes). Figure 3 shows the result of fitting of the DHO model and CDHO model to the data.

Table III and Table IV show the values of the fitting parameters, for damped harmonic oscillations model and critically damped harmonic oscillations model, respectively.

Table III summarizes parameters obtained through non-linear numerical fit of the DHO model to time-series of housing prices under study. According to that data, delay shift remains in a narrow range between 5 and 6 regardless of the market, whereas significant differences are found in the relaxation constant $\tau$. In this light, the housing market in Warszawa turns out the most inert 


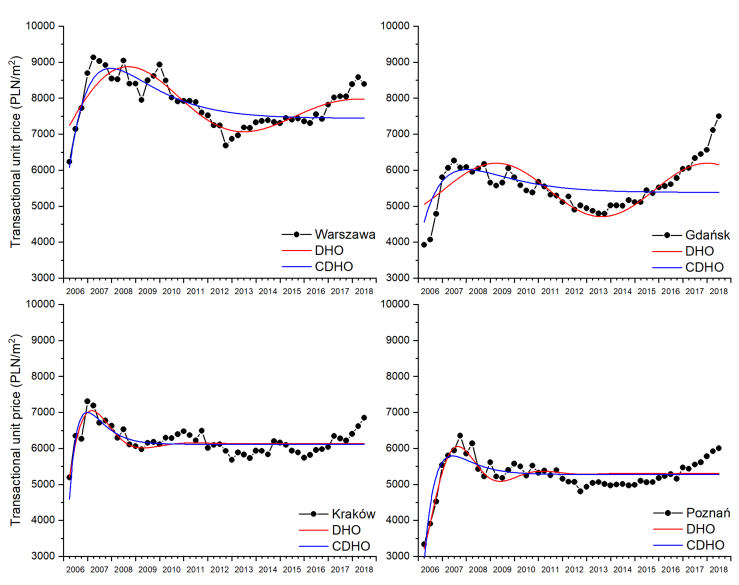

Fig. 3. Results of numerical fit processing of DHO and CDHO models to time series of quarterlyaveraged transactional housing prices in 4 polish cities in period 2006-2018.

TABLE IV

Dynamical properties of housing market obtained through fit of time-series of housing prices to critically damped harmonic motion model (CDHO).

\begin{tabular}{l|c|c|c|c}
\hline \multicolumn{1}{c|}{ Town } & $t_{0}$ [quaters] & $\tau$ [quaters] & $y$ [PLN] & $R^{2}$ \\
\hline Warszawa & 441 & 538 & 744348 & 0.683 \\
Gdańsk & 2.83 & 536 & 538350 & 0.219 \\
Kraków & 288 & 211 & 611593 & 0.913 \\
Poznań & 4.21 & 2.65 & 527812 & 0.790 \\
\hline
\end{tabular}

The procedure of fitting of all time-series of housing prices to damped harmonic motion model was reached, for chi-square with value of $10^{-9}$

(the highest relaxation constant), whereas that in Kraków - the most volatile. Warszawa also stands out from the rest in terms of the longest oscillation period $T$ and the largest equilibrium prices $y_{0}$.

On the other hand, Table IV presents the results of similar fit procedure using the CDHO model. As in DHO model, delay shifts appeared nearly constant among the time-series, and similar observation can be drawn upon analysis of the relaxation constants. Unlike the DHO model, the CDHO model exhibits only slightly larger inertness of the housing prices in Warszawa with respect to other markets, but supports the claim on the volatility of the market in Kraków. Equilibrium prices obtained thanks to CDHO model are nearly the same as in previous model.

Coefficient of determination ( $R$-square ) calculated in the framework of fitting a damped harmonic motion model (Table III) and critically damped harmonic motion model (Table IV) to housing prices indicated different levels of linear relationship between two variables. $R^{2}$ coefficient for the DHO model, from 0.558 to 0.728 were obtained, and from 0.219 to 0.913 for CDHO model. In housing market research, as an area of the social sciences, we have to consider that $R^{2}$ is a measure of explanatory power, not fitting. In particular, the coefficient of determination $R$-square is a relative measure, consisting of comparing the error rate associated with the model under analysis and the error rate associated with the reference model (in this case the average model) [18]. Hence, it is a relative, not an absolute measure of model quality. According to Woolridge [19], it is therefore not possible to establish a definitive criterion for the value of the coefficient $R^{2}$ (for example 0.9) where the model can be considered "good". Regression models, especially in the social or behavioural sciences, will by nature not include all relevant predictors explaining the resultant variable. In some disciplines the $R$-square is usually higher because it is easier to identify complete, well-defined models. In the social sciences, where it is difficult to determine a complete set of explanatory variables, relatively low $R$-square values are often expected. The housing market is an imperfect market, where behavioural factors may play a key role, and it is difficult and sometimes even impossible to incorporate such factors into the model. Therefore, in most research papers, the determination coefficient of $0.6-0.8$ in models describing housing market prices is considered to be satisfactory, for example 0.61 [20], 0.74 [21], 0.84 [22], $0.77-0.83$ [23].

The critically damped harmonic motion model has reached miscellaneous results of determination coefficient, from 0.219 (Gdańsk) to 0.913 (Kraków). The damped harmonic motion model shows more commensurable results, from 0.558 (Kraków) to 0.728 (Warszawa). Because at every plot (Fig. 3) the fitted function starts to diverge from the data at the end of the time window, we decided to find the time periods for which the DHO model estimation would give the highest $R^{2}$ value. Initially, we thought we were dealing with further oscillations, and that after the end of the period of growth there would be a price decrease. We conducted the fitting from the DHO model with degressively smaller periods than originally studied, i.e., 2006-2018. Table V shows the values of determination coefficients calculated in the fitting process of the time series of housing prices to damped harmonic motion model (DHO) by periods. Figure 3 shows the result of fitting the damped harmonic motion model to the data (2006-2014 period).

The results in Table $\mathrm{V}$ indicate that the highest value of $R$-square is obtained for the period 2006-2014. This may indicate a paradigm change in the housing market dynamics. the previous model, of a sharp rise in prices in 2006-2007 followed by slow decreases in prices in the form of declining oscillations, is turning into a completely different trend. Concurrently, Fig. 4 shows a better fit of DHO model to time series of dwelling prices from Warszawa, Gdańsk, Kraków and Poznań. It can therefore be concluded that beginning in 2014 there has been a permanent change in the direction of the price dynamics of dwellings. It can be assumed that this is the beginning of another cycle of economic boom on this market. 
TABLE V

The determination coefficients obtained through fit of time-series of housing prices to damped harmonic motion model (DHO) by periods.

\begin{tabular}{c|c|c|c|c|c}
\hline \hline \multirow{2}{*}{ Town } & \multicolumn{5}{|c}{ The determination coefficient $R^{2}$} \\
\cline { 2 - 6 } & $2006-2017$ & $2006-2016$ & $2006-2015$ & $2006-2014$ & $2006-2013$ \\
\hline Warszawa & 0.783 & 0.829 & 0.833 & 0.836 & 0.834 \\
Gdańsk & 0.652 & 0.799 & 0.854 & 0.880 & 0.876 \\
Kraków & 0.662 & 0.682 & 0.686 & 0.687 & 0.683 \\
Poznań & 0.803 & 0.812 & 0.843 & 0.849 & 0.842 \\
\hline
\end{tabular}

The procedure of fitting of all time-series of housing prices to damped harmonic motion model was reached, for chi-square with value of $10^{-9}$

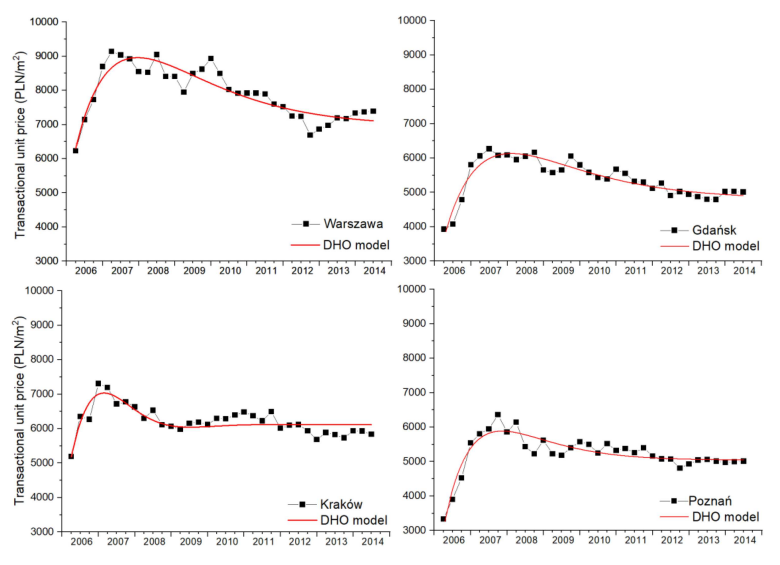

Fig. 4. Results of numerical fit processing of DHO model to time series of quarterly-averaged transactional housing prices in 4 polish cities in period 2006-2014.

In addition, in our study we have conducted a procedure to fit classic non-linear functions. We wanted to compare the value of DHO determination coefficient of the model for the time series of dwelling prices in Gdańsk $\left(R^{2}=0.88\right)$ with other non-linear functions. For all analysed functions the procedure of fitting was reached, with a chi-square value of $10^{-9}$. We have obtained the following results of $R$-square : Boltzmann Function (0.26); Lorentzian peak function with bell shape and much wider tails than Gaussian function (0.52); Area version of Gaussian Function (0.54); Amplitude version of Gaussian peak function (0.544); Sine wave function oscillates around a specified value (0.57); Bi-Gaussian peak function used to fit asymmetric peak (0.588). Neither of the adopted non-linear models exceeded the value of 0.6 determination coefficient at $R$ square equal to 0.88 damped harmonic motion model (DHO).

\section{Conclusions}

This paper presents results of interdisciplinary research, which explores the benefits of applying the methods specific of physical sciences into socioeconomic geography (spatial economy) as a part of economy science. the inertia of housing markets becomes manifested during periods of sharp rises or sudden falls in prices, when they are subjected to deep changes of structural origin, whereas the quasistationary long-term evolution is slow enough to ensure a state of system equilibrium.

Both DHO and CDHO models exhibit significant inertia of the local housing markets in terms of large relaxation constant $(\sim 1$ year $)$. From the DHO model, the oscillation period (the length of the economic cycle) is found to vary between 3 and 10 years. Apart from relaxation processes caused by "damping", local housing markets remain under temporarily activated influence of external factors that contribute to housing price bubbles. The model of damped harmonic motion helps to exhibit dynamical properties of local housing markets in Warszawa, Kraków, Poznań and Gdańsk. Relative delays in onsets of sharp changes of the prices point at the dominant market (Warszawa) in the system supporting the mechanistic idea of the system of communicating vessels influenced by various stimulations of external and internal origins. The calculations show that the Warsaw market is apparently a link in the transfer of impulses from the world economy to other housing markets in Poland.

The study also shows that the highest fitting levels of the DHO model are obtained for the time period 2006-2014, when there was a permanent process of changing the direction of price dynamics (the beginning of a new cycle of the housing market). the damped harmonic motion model showed higher matching measures than did other non-linear models.

\section{References}

[1] G. Galster, Urban Stud. 33, 1797 (1996).

[2] C. Leung, J. Hous. Econ. 13, 249 (2004).

[3] Z. Adams, R Füss, J. Hous. Econ. 19, 38 (2010).

[4] J. Batóg, I. Foryś, R Gaca, M. Głuszak, J. Konowalczuk, Sustainability 11, 412 (2019). 
[5] J. Yates, M. Berry, Hous. Stud. 26, 1133 (2011).

[6] S. Moulton, J. Hous. Econ. 24, 21 (2014).

[7] E. Siemińska, M. Rymarzak, Real Estate Manage. Valuat. 22, 68 (2015).

[8] N. Yoshino, F. Taghizadeh-Hesary, Int. J. Monet. Econ. Finance 7, 157 (2014).

[9] S. Chatterjee, B. Eyigungor, Rev. Econ. Dyn. 18, 165 (2015).

[10] J.E. Stiglitz, Am. Econ. Rev. 92460 (2002).

[11] A. Jakimowicz, S. Kulesza, Acta Phys. Pol. A 133, 1351 (2018).

[12] S. Kokot, Real Estate Manage. Valuat. 22, 14 (2014).

[13] I. Dittmann, Real Estate Manage. Valuat. 21, 39 (2013).

[14] K. Żelazowski, Real Estate Manage. Valuat. 27, 44 (2019).
[15] S. Kulesza, M. Bełej, Acta Phys. Pol. A 127, A-99 (2015).

[16] A. Jakimowicz, Acta Phys. Pol. A 129, 897 (2016).

[17] M. Bełej, Real Estate Manag. Valuat. 26, 22 (2018).

[18] N. Zaarour, E. Melachrinoudis, J. Educ. Train. Stud. 7, 27 (2019).

[19] J.M Wooldridge, Econ. Lett. 36, 49 (1991).

[20] M. Hannonen, Int. J. Strateg. Prop. Manag. 9, 145 (2005).

[21] J. Benjamin, R. Guttery, C.F. Sirmans, J. Real Estate Pract. Educ. 7, 65 (2004).

[22] B.J. Sopranzetti, in: Handbook of Quantitative Finance and Risk Management, Eds. CF. Lee, A.C. Lee, J. Lee, Springer, Boston (MA) 2010, p. 1201.

[23] A.M. Yusof, S. Ismail, Commun. IBIMA 2012, 1 (2012). 